\title{
Design of a dynamic and self-adapting system, supported with artificial intelligence, machine learning and real-time intelligence for predictive cyber risk analytics in extreme environments - cyber risk in the colonisation of Mars
}

\author{
Petar Radanliev ${ }^{1}$ (D) David De Roure ${ }^{1} \cdot$ Kevin Page $^{1} \cdot$ Max Van Kleek $^{2} \cdot$ Omar Santos $^{3} \cdot$ La'Treall Maddox $^{3}$. \\ Pete Burnap ${ }^{4}$. Eirini Anthi ${ }^{4}$. Carsten Maple ${ }^{5}$
}

Received: 1 November 2020 /Revised: 2 December 2020 / Accepted: 13 January 2021 / Published online: 10 February 2021

(C) The Author(s) 2021

\begin{abstract}
Multiple governmental agencies and private organisations have made commitments for the colonisation of Mars. Such colonisation requires complex systems and infrastructure that could be very costly to repair or replace in cases of cyber-attacks. This paper surveys deep learning algorithms, IoT cyber security and risk models, and established mathematical formulas to identify the best approach for developing a dynamic and self-adapting system for predictive cyber risk analytics supported with Artificial Intelligence and Machine Learning and real-time intelligence in edge computing. The paper presents a new mathematical approach for integrating concepts for cognition engine design, edge computing and Artificial Intelligence and Machine Learning to automate anomaly detection. This engine instigates a step change by applying Artificial Intelligence and Machine Learning embedded at the edge of IoT networks, to deliver safe and functional real-time intelligence for predictive cyber risk analytics. This will enhance capacities for risk analytics and assists in the creation of a comprehensive and systematic understanding of the opportunities and threats that arise when edge computing nodes are deployed, and when Artificial Intelligence and Machine Learning technologies are migrated to the periphery of the internet and into local IoT networks.
\end{abstract}

Keywords Dynamic and self-adapting systems · Artificial intelligence $\cdot$ Machine learning $\cdot$ Real-time intelligence $\cdot$ Predictive cyber risk analytics $\cdot$ Colonisation of Mars $\cdot$ Cyber-risk analytics in extreme environments $\cdot$ Cyber-risk in outer space

\section{Introduction}

The advancements of artificial intelligence in industrial automation, triggers questions on safety and security, and whether

Petar Radanliev

petar.radanliev@oerc.ox.ac.uk

David De Roure

david.de.roure@oerc.ox.ac.uk

Max Van Kleek

m.d.c@oxford.ac.uk

Omar Santos

oma.rsantos@cisco.com

La'Treall Maddox

latrea.1lmadox@cisco.com

Pete Burnap

p.b.urnap@cs.cardiff.ac.uk
AI has enhanced security or increased the cyber risks in extreme environments (Khan 2020), This is specifically of concern in some extreme environments where cyber-attacks can cause significant and irreversible damage, such as the space

Eirini Anthi

e.ant@cs.cardiff.ac.uk

Carsten Maple

k.m.c@warwic.ac.uk

1 Department of Engineering Sciences, University of Oxford, Oxford e-Research Centre, 7 Keble Road, Oxford OX1 3QG, UK

2 Department of Computer Science, University of Oxford, Oxford, UK

3 Cisco Research Centre, Research Triangle Park, Raleigh-Durham, NC, USA

4 School of Computer Science and Informatics, Cardiff University, Cardiff, UK

5 MG Cyber Security Centre, University of Warwick, Coventry, UK 
exploration industry. Commitments on the colonisation of Mars have emerged from multiple governmental agencies (e.g. NASA, ESA, Roscosmos, ISRO and the CNSA) and private organisations (e.g. SpaceX, Lockheed Martin, and Boeing). The colonisation of Mars presents many difficulties and hazards, such as radiation exposure, toxic soil, low gravity, lack of water, cold temperatures, and social isolation. One specific risk has been ignored until now, the risk of cyberattacks. Since the colonisation of space requires the reinstalment of critical infrastructure, and such infrastructure needs to be installed by smart machines, then we need to anticipate that the risk assessment of such smart machines operating autonomously, should be undertaken by other smart machines. We also need to anticipate the cost (including the cost of down time) required for repairing or replacing such machine - in outer space. This cost (and cost of down time) should be understood prior to deciding on the most appropriate space systems. Although such systems can be tested prior to deployment, the risk assessment after deployment would be completely reliant on Artificial Intelligence (AI), because there will be no human participation in the installation phases.

In this paper, we conduct a survey review of different Artificial Intelligence and Machine Learning (AI/ML) methods, that could be used for the risk assessment after deployment. Recent studies on Artificial Intelligence and Machine Learning (AI/ML) perspectives on mobile edge computing lack detail (Radanliev et al. 2020d), but provide guidance on how data can be processed in real-time, reducing edge-cloud delay and inform on the topic of cognitive cyber security at the edge. Since the risk assessment of deployed space systems is extremely difficult, due to the extreme conditions, this paper is focused on the topic of predicting cyber risk loss magnitude through dynamic analytics of cyber-attack threat event frequencies. Forecasting the threat events, could enable preventing such events from occurring in the first place. Secondly, we investigate what data is required for applying $\mathrm{AI}$ algorithms in dynamic risk analytics. Additional challenges addressed in this study relate mainly to socio-technical issues, such as technology, regulation, supply chains and control systems. For example, investigating the perceptions of risk and trustworthiness that emerge as a result of machine agency, which interact with regulation, standards and policy on the one hand and design and engineering on the other, spanning the physical and behavioural sciences. But the specific focus of this paper is on integrating $\mathrm{AI} / \mathrm{ML}$ in the data collection and analytics of risk through fog computing (i.e. use of edge devices) for forward-facing predictive outputs. We investigate a scenario where an organisation planning for colonisation of outer space, has implemented all the security recommendations (e.g. NIST), but the risk remains from uncertain and unpredictive attack vectors in outer space, at the edge of the network.
For narrowing the topic to assessment of these new types of cyber security, the research adopts a red teaming methodology for detecting and reducing threats and simplify compliance with internal, industry and government regulations. A red teaming approach is firstly applied by challenging plans, policies, systems and assumptions and adopting an adversarial approach to IoT cyber risks. With this approach, IoT cyber risks can be divided in three levels, edge, fog and cloud. The fog computing is placed in the distribution network layer and provides sufficient computational resources, low latency and compute-intensive applications. The cloud computing level represent a shared pool of rapidly provisioned computing resources, for high computation and data storage. Hence, IoT cybersecurity deployment in the fog and cloud computing levels is not a big concern. The small computation capability at the edge devices makes IoT cyber risk more likely to occur at the edge computing level. Hence, this article is primarily focused on the edge computing level.

Since the focus of this review was the risk at the edge of the network, we applied this as the basis of selecting the AI methodologies considered in Table 1. However, there are many techniques that are not discussed in this review. A holistic review of all $\mathrm{AI}$ methodologies was considered beyond the scope of this review, and since many AI methodologies are not directly relevant to this topic, we selected only the most appropriate AI methodologies. To select the most appropriate AI methodologies, we applied a red teaming approach to identify IoT systems that are mostly affected by a few types of network risk event. Those include: Eavesdropping Attacks, Denial of Service (DoS) and Distributed DoS (DDoS), Spoofing Attacks, and Man-in-the-Middle attacks (MITM). To describe briefly the relationship between these types of attacks, Eavesdropping Attacks is used for listening IoT communications without the transmission appearing abnormal, hence making it difficult to detect. After Eavesdropping Attacks has gained authorisation access, Spoofing Attacks are used to send spoofed traffic with a legitimate access to IoT network. The MITM is just an advanced Spoofing Attack where adversary is positioned between two IoT devices and independently intercepts data and communicates between endpoints, collecting sensitive information, dropping packets, and causing different security vulnerabilities. The DoS and DDoS floods the IoT devices network with traffic, this overloads the communication and exhausts the network, leading to IoT devices being unable to communicate. As simple as it is, this is the most common and most dangerous IoT attack. The small computation capability at the edge devices, make DDoS attacks really difficult to resolve. While new cyber security is constantly been developed (e.g. ISO 3000), the level of cyber-attack sophistication is also increasing (NetScouts 2018) (e.g. the Mirai variants 'VPN filter' is delivered in multiple stages with modularised payload; 'TORii' uses its own encryption and evasion tactic). Considering these 
Table 1 AI/ML algorithm application for descriptive, predictive, and prescriptive risk analytics in edge computing

\begin{tabular}{|c|c|c|}
\hline $\mathrm{AI} / \mathrm{ML}$ technique & Application & References \\
\hline Deep learning - ANN & Network architecture & $\begin{array}{l}\text { Berman et al. (2019), Diro and Chilamkurti (2018), } \\
\text { Roopak et al. (2019), Vinayakumar et al. (2019) }\end{array}$ \\
\hline $\begin{array}{l}\text { Anomaly detection, unsupervised learning, } \\
\text { classification }\end{array}$ & \multirow[t]{2}{*}{ Network planning/load balancing } & Gebremariam et al. (2019), Ullah et al. (2019) \\
\hline Regression & & Hu et al. (2017) \\
\hline Classification - Bayesian networks & \multirow{2}{*}{$\begin{array}{l}\text { Fault and failure detection/ } \\
\text { management }\end{array}$} & Bashir et al. (2019), Sultana et al. (2019) \\
\hline Classification/Clustering - autoencoders & & Al-Turjman (2020) \\
\hline $\begin{array}{l}\text { Algorithms: supervised, unsupervised and } \\
\text { reinforcement learning }\end{array}$ & \multirow[t]{6}{*}{$\begin{array}{l}\text { Network management and } \\
\text { operations }\end{array}$} & Cui et al. (2019), Nguyen et al. (2019) \\
\hline ANN (RNN) and random forest & & Park et al. (2018) \\
\hline Regression - ANN & & Anagnostopoulos and Hadjiefthymiades (2019) \\
\hline Classification - Naive Bayes (NB) & & (Syafrudin et al. (2018), Yin et al. (2019) \\
\hline $\begin{array}{l}\text { Classification algorithms: K nearest } \\
\text { neighbours, SVM }\end{array}$ & & $\begin{array}{l}\text { Guo et al. (2018), Sangaiah et al. (2019), } \\
\text { Zhang et al. (2019) }\end{array}$ \\
\hline GDNN & & J. Wang et al. (2019a), X. Wang et al. (2019b) \\
\hline ANN & \multirow[t]{5}{*}{ Network security and breach detection } & Sun et al. (2019) \\
\hline Dynamic game - Nash Folk theorem & & Abegunde et al. (2016) \\
\hline Game theory and NB classifier & & Bui et al. (2017), Moura and Hutchison (2019) \\
\hline Deep learning algorithms & & Blanco-Filgueira et al. (2019), Li et al. (2018) \\
\hline $\begin{array}{l}\text { Algorithms: supervised, unsupervised and } \\
\text { reinforcement learning }\end{array}$ & & Cao et al. (2019) \\
\hline
\end{tabular}

continuous changes, to assess the effectiveness of cybersecurity, we need cyber analytic approaches that can handle real time intelligence in the form of probabilistic data collected at the edge. But the effectiveness of cybersecurity should not only be measured by the protection of cyberspace, but also with the protection of assets that can be reached via cyberspace (Davis et al. 2019).

In brief, we investigate the role of $\mathrm{AI} / \mathrm{ML}$ in cyber risk analytics with use of confidence intervals and time bound ranges at the edge. The objective of such an approach would be to protect data integrity, while securing predictive analytic outputs and integrating such solutions in these new types of edge computing cyber security. In edge computing, the IoTaugmented physical reality is open to adversarial behaviours that are yet uncharted and poorly understood, especially the socio-technical dimensions. This paper evaluates the impact of compromise in terms of its safety implications and resulting consequences on end system provision.

\section{Research methodology}

The research methodology applied consist of a survey review investigating different deep learning and machine learning algorithms and their application in AI for securing the edge. The survey review is used for investigating the intersections between cyber risk and technology, regulatory interventions, and economics.

The research methodology in this was survey review paper, was developed to address the (un)availability of data. Although there is a valid concern about the (un)availability of data, in the present digital age, the IT and IoT devices create a large volume of data. Hence, the real challenges that remain, are in developing suitable data strategies to utilise this new data. Simultaneously, the cyber security architecture for complex coupled systems, demands data strategy optimisation and decision making on collecting and assessment of probabilistic data. With consideration of the above, the research integrates impact assessment models, with AI and risk analytics models, for developing a dynamic and self-adopting data analytics methodology to assess, predict, and analyse cyber-risks.

For risk assessment of systems for colonising outer space, we need a quantitative risk impact estimation is needed - for estimating cyber security and cyber risk at the edge (Radanliev et al. 2020e). Our argument is that without a dynamic real-time probabilistic risk data and cyber risk analytics enhanced with AI/ML, these estimations can be outdated and imprecise. We are concerned not just with securing a system, but to acknowledge that failure and compromise will occur and address how the system responds in these circumstances. This is an important methodological principle which distinguishes out work within the cybersecurity domain. Recent 
literature confirms diverse cyber risks from IoT systems (Maple 2017), including risks in IoT ecosystems (Tanczer et al. 2018) and IoT environments (Breza et al. 2018), such as risk from smart homes (Eirini Anthi et al. 2019; Ghirardello et al. 2018), the Industrial IoT (Boyes et al. 2018), and challenges in security metrics (Agyepong et al. 2019). Cybersecurity solutions for specific IoT risks are also emerging at a fast rate, such as new models on opportunities and motivations for reducing cyber risk (Safa et al. 2018), adaptive intrusion detection (E. Anthi et al. 2018), security economic by design (Craggs and Rashid 2017), highlighting the privacy requirements (Anthonysamy et al. 2017) and strategies for achieving privacy (Van Kleek et al. 2018). Therefore, our methodology is based on mathematical principles and quantitative data. In recent publications on this topic (Radanliev et al. 2020b), we discovered that the lack of probabilistic data leads to qualitative cyber risk assessment approaches, where the outcome represents a speculative assumption. Emerging quantitative models are effectively designed with ranges and confidence intervals based on expert opinions and not probabilistic data (Buith 2016).

\section{Survey of $\mathrm{Al} / \mathrm{ML}$ algorithms}

The AI/ML are essential for advancing beyond the limitations of Value-at-Risk (VaR) models (FAIR 2017), where Bayesian and frequentist methods are applied with and beyond VaR models (Malhotra 2018). This requires federated learning and blockchain based decentralised AI architecture where AI processing shifts from the cloud to the edge and the AI workflow is moved and data restricted to the device (Porambage et al. 2019). Current gaps in cyber risk analytics are in the areas of descriptive, predictive, and prescriptive analytics (Barker et al. 2017). Hence, a survey of AI/ML applications is presented in Table 1, to address the main questions emerging from this study on edge computing and descriptive, predictive, and prescriptive risk analytics.

Table 1 confirms that by integrating AI/ML in the risk analytics, we can devise a new approach for cognitive data analytics, creating a stronger resilience of systems through cognition in their physical, digital and social dimensions. This approach resolves around understanding how and when compromises happen, to enable systems to adapt and continue to operate safely and securely when they have been compromised. Cognition through $\mathrm{AI} / \mathrm{ML}$ and how cognitive real time intelligence would enable systems to recover and become more robust is evaluated in more detail below. The survey in Table 1 is informed by but avoids overlapping with a series of working papers and project reports on IoT cyber risk, IoT risk assessment and IoT at the edge found in pre-prints online. This research is specifically focused on AI/ML in IoT risk analytics, and it benefits from this established research knowledge. But with a focus on the topic of securing the edge through AI/
ML real time analytics to build stronger transformative and impactful understanding on the topic.

Majority of the current Intrusion Detection Systems (IDS) are based on ML algorithms and the CNN (Convolutional Neural Network) + LSTM (Long Short Term Memory) appear to perform better than other Deep Learning (subsets of ML) models (Roopak et al. 2019). Such arguments are difficult to generalise when tested with a single dataset. Deep Neural Network (DNN) has been applied with distributed deep learning to collect network-based and host-based intrusion detection systems (NBID and HBID) (Vinayakumar et al. 2019). This is a very comprehensive study, where a multilayer perceptron (MLP) model is adopted. However, in a related research, the MLP (type of artificial neural network - ANN) was found to be the least accurate deep learning model (Roopak et al. 2019).

Network Based Intrusion Detection Systems (NIDS) that use statistical measures or computer thresholds have been related to security research since the early days of computer architecture (Vinayakumar et al. 2019). But are ineffective for current cyber risk analytics of connected and highly complex ICT systems, because they present high rates of false negatives (failure to detect) and false positives (false alerts). Distributed attack detection at fog level was proven to be more scalable than centralised cloud for IoT (Diro and Chilamkurti 2018). If the attack vectors are known, then up to $99.999 \%$ accuracy can be reached by type of attack with bidirectional long short-term memory (LSTM) units introduced to recurrent neural network (RNN) (Berman et al. 2019). Similarly, a Siamese Network Classification Framework (SNCF) can alleviate imbalance in risk prediction and present more reliable results when compared with other algorithms (Sun et al. 2019). With SNCF two different types of risk data sets can be used, (1) public data set (less features and more samples), (2) real data set (more features and less samples). The first set could verify solving the imbalance problem, and the second could eliminate reliance on the characteristics of feature engineering. Such experimental SNCF results have shown good cyber risk prediction performance (Sun et al. 2019) and Software Defined Networking Technology (SDN) has been effective in detecting and monitoring network security when integrated with Machine Learning (ML) and deep learning (DL) to create SDNbased NIDS (Sultana et al. 2019). The main risk concern with SDN and Network Functions Virtualisation (NFV) is the centralised nature which creates a single point of failure (Gebremariam et al. 2019). To resolve this, three layered nodes (Edge-IDS, Fog-IDS, and Cloud-IDS) has been proposed for NIDS system in SDN-based cloud IoT networks (Nguyen et al. 2019). Cloud environments enable IoT device virtualisation resulting with virtual IoT objects that can be accessed and controlled remotely though a dynamic virtual network (Ullah et al. 2019). 
A power load forecasting (Hu et al. 2017), can be based on the generalised regression neural network with decreasing step fruit fly optimisation algorithm. Similarly, logistic regression and multicriteria decision making in IoT fog computing can be used for resource allocation (Bashir et al. 2019). The main concern we have about the development of such algorithms is that deployment of $5 \mathrm{G}$ can separate real-time intelligence and security between IoT, IoE or even IoNT (Al-Turjman 2020). Hence, intelligence and cognition techniques would differ in application areas and architecture. One of the possible issues is that $\mathrm{ML}$ platforms (such as TensorFlow, Gaia, Petuum, Apache Spark, and GraphLab), are designed for offline data analytics and training data are collected, partitioned, and learned offline to construct machines for data analytics (Cui et al. 2019). While some of the recently proposed detection systems for edge computing are operating in real time, e.g. LiReD (Park et al. 2018). Edge nodes can host and process the data to limit latency, and recently enhanced models can handle the earlier problems with missing values (Anagnostopoulos and Hadjiefthymiades 2019), while improving the detection accuracy (Yin et al. 2019) and decision making with early warning systems (Syafrudin et al. 2018). The classification accuracy can also be improved with edge filtering (Guo et al. 2018), position confidentiality (Sangaiah et al. 2019) and dynamic data classification (Zhang et al. 2019), to avoid system overload when tasks increase suddenly, by diverting and allocating complex tasks to devices with stronger computing power.

Multi-Access Edge Computing based on reinforcement learning, enhances the performance of such 'offloading' in polynomial time complexity - worst-case running time (J. Wang et al. 2019a). While integration of Deep Reinforcement Learning and Federated Learning with mobile edge systems, optimises mobile edge computing, caching, and communication, and makes edge systems more intelligent (X. Wang et al. 2019b). Optimising and balancing resource constrains in edge computing has been investigated with 'dynamic game'(Abegunde et al. 2016) and 'game theory' (Moura and Hutchison 2019) strategies. Such optimisation is primarily theoretical, but highly relevant for red teaming of edge computing risks. Two models 'Cournot' and 'Stackelberg' are proposed for making real-time optimisation of traffic flow (Bui et al. 2017). These models need to be tested with real-time data to be verified, but the theoretical contribution is quite significant, e.g. applying the 'Prisoners Dilemma' on optimising decisions.

Deep learning models recorded highest accuracy as $97.16 \%$ detection of DDoS attacks (Roopak et al. 2019), and the multilayered structure, makes them very adoptable to edge computing. Hence, deep learning has been applied for optimising performance while protecting user privacy in uploading data, $(\mathrm{Li}$ et al. 2018). But the computing and memory requirements, along with the high power consumption, make them difficult to use in edge computing (Blanco-Filgueira et al. 2019). Further research is needed to identify how deep learning can be applied in practice, with real-time data. Possibly reinforcement learning, supervised/unsupervised learning, and deep reinforcement learning (Cao et al. 2019), would provide some insights into how this can be achieved.

\section{Elements of artificial intelligence and machine learning in cognition engine design}

Cyber risk analytics at present is reactive and assessments are based on risk/loss events that already occurred (Radanliev et al. 2020a). AI/ML in forward-looking predictive analytics enable threat intelligence prediction and faster attack detection. The main advantage of AI in risk analytics is the fast processing and analysis of big data where parsing, filtering and visualisation is done in near real time (Radanliev et al. 2020c). Machine learning uses mathematical and statistical methods and algorithms that learn, build and improve models from data. This enables design of a cognition engine in the form of automated predictive cyber intelligent software agents that identify, assess and record cyber-attacks. After this, natural language processing (NLP) can be applied to perform behaviour analytics and create baseline profiles of normal behaviour and then monitor for abnormalities while continuously learning from the profile's behaviour patterns. Facilitating a consistent and repeatable detection of threat indicators and predictions about new persistent risks that are undetected. AI/ML learn from multiple patterns (e.g. threat intelligence feed, device event logs, vulnerability information, contextual data) to determine predictive risk insights. Predictive risk analytics for advance notice of risk exposure and potential loss can be performed through monitoring the risk lifecycle activities, e.g. the reactive activities that capture losses and near miss events. From reactive activities we can quantify the impact of losses and develop baseline indicators to compare mathematical results.

\section{Mathematical formulae}

To develop predictive risk analytic methodology for estimating the loss of cyber risk, we apply adapted version of the aggregate loss method to compound a Poisson discrete probability distribution. For the adopted version, we use the theoretical cumulative distribution function of aggregate loss, as shown in (Charpentier 2014):

$$
F_{L_{c}}(l)=\sum_{n=0}^{+\infty} P(N=n) P\left(\sum_{i=1}^{n} Z i<l\right)
$$

In the adapted version, we generate the frequency distribution from the cumulative function in Eq. 1, with non-linear 
summation and simulated random variables to approximate the theoretical function.

The $L \mathscr{C}=$ aggregate loss distribution consisting of the compound sum of $N=$ frequency (intensity) and $Z \mathscr{I}=$ severity (loss) distribution and is described as: $L \mathscr{C}=\sum_{i=1}^{N} Z \mathscr{I}$, where $L \mathscr{C}=0$, if $N=0$.

Considering the (un)availability of probabilistic data, the $N$, $Z \mathscr{I}$, and the consequent $Z j$ where $(\mathscr{I} j)$ are considered independent. This cumulative function defines a frequency distribution for aggregate loss as nonlinear summation. The function can be improved by considering the frequency distribution as Poisson variable, where for a given time interval $\left[0, t_{\mathrm{c}}\right]$, the inter-arrival time $=S_{\mathrm{i}}$ of two 'risks' within the interval follows an exponential distribution with parameter $\lambda_{\mathrm{c}}$. This function can be described as:

$$
\begin{aligned}
& P(N(t)=n)=\frac{\exp \left(-\lambda_{c} t_{c}\right)\left(\lambda_{c} t_{c}\right)}{n !} \\
& Y_{i}=S_{i}-S_{i-1} \sim \operatorname{Exp}\left(\lambda_{c}\right), F(Y<y)=1-\exp \left\{-\lambda_{c} y\right\}
\end{aligned}
$$

The known issues with (un)availability of sufficient probabilistic data (Radanliev et al. 2018) can be mitigated by enhancing the precision of the sample size in the inter-arrival time, where the insufficient (few years) data can be considered as lognormal (Galton) distribution where $t_{c}=365$ (representing 365 days). In a more specific dataset scenarios, the distribution will vary depending on the probabilistic data. We postulate that the $t_{c}=365$ has a fixed loss per day $=b$, where $M_{x}=$ total loss days for an IoT device $I o T_{x}$ and the device is operational at time $t$ and the total loss per $t_{c}=b$ $\times M_{x}$. Considering that IoT device can stop functioning (or be killed by grey-hat attack) at any point in $t_{c}$, then $M_{x}$ represents a continuous random variable of the future (potential) loss from an IoT device infected for time $x$ and $1_{(.)}$, with a given discount rate $=r$ calculated as $v=(1+r)^{-1}$, then considering the probability of IoT device stops (or be killed) and the discount factor, the present potential loss $P_{x}$ can be determined as:

$P_{x}=v \times 1_{\left(T_{x}>1\right)} \times\left(b \times M_{x}\right)$

This formula calculates the risk of loss depending on the IoT device surviving the entire 365 days, or stops (or be killed) during the 365 days. The second postulate is that the risk of loss is eliminated when the device is killed. The actuarial equivalent of this can be explained as the present values of the expected losses described as loading $=\delta$ and expected revenue (that was lost) $=\pi_{\chi}$ are equal to:

$\pi_{\chi}=(1+\delta) \times E\left(P_{x}\right)$

In time, when more extensive data from IoT devices becomes available, more precise $\delta$ can result with lowering the expected loss $=\alpha$ and a more precise expected present value of the loss $=E\left(P_{\chi}^{1}\right)$ can be estimated as:

$$
E\left(P_{\chi}^{1}\right)=\propto \times E\left(P_{x}\right)
$$

and expected revenue (that was lost) as:

$$
P_{\chi}^{1}=(1+\delta) \times E\left(P_{\chi}^{1}\right)
$$

The continuous random variable of the future (potential) loss $M_{x}$ can be divided on the number of attacks (frequency) $=N_{x}$ and the and severity (loss) $=Z \mathscr{I}$ per breach in a given $t_{c}$ can be denoted as $R_{x, i}$ and $N_{x}$ would reflect a Poisson distribution with time-varying intensity $\theta_{\chi}$, and $R_{\chi, 0}$ and $R_{\chi, 1}=\Upsilon_{\chi}$, ${ }_{1}+1$ where $\Upsilon_{\chi, 1}$ follows time-varying intensity $=\lambda_{\chi}$. The $M_{x}$ in a given $t_{c}$ for an IoT device $I o T_{x}$ can be estimated as:

$$
M_{x}=\sum_{i=o}^{N_{\chi}} R_{x, i}
$$

With a compound Poisson process, the probable present potential loss $P_{x}$ with a given $M_{x}$ in a given $t_{c}$ for an IoT device $I o T_{x}$, can be calculated with:

$$
P\left(M_{x}=n\right)=\left\{\begin{array}{c}
\exp \left(-\theta_{\chi}\right), n=0 \\
\sum_{j=1}^{n} \frac{j\left(\lambda_{\chi}\right)^{n-j}\left(\theta_{\chi}\right)^{j} \exp -\left(j \lambda_{\chi}+\theta_{\chi}\right)}{\begin{array}{l}
j !(n-j) ! \\
n \geq 1
\end{array}},(9)
\end{array}\right.
$$

The above equation is designed for IoT risk assessment, but it can easily be adopted for different types of cyber risks. For example, we could calculate IoT cyber risk from AI as $\kappa$ for a given IoT device $I o T_{x}$ with $\kappa \times \pi_{\chi}^{1}$, where in the first instance, the total loss $L_{\kappa}$ would include $M_{x}$ and $L \mathscr{C}$. This can be expressed as:

$$
L_{\kappa}=\sum_{j=1}^{k} M_{x}+L \mathscr{C}
$$

and evaluated with risk proxies from shortfall probability, Value at Risk and Conditional Tail Expectation. The shortfall probability can be calculated as: 


$$
\operatorname{Prob}(\operatorname{Shortfall})=\operatorname{Prob}\left(\kappa \times \pi_{\chi}^{1} \leq L_{\kappa}\right)
$$

where expected shortfall is:

$$
E(\text { Shortfall })=E\left(\max \left(\kappa \times \pi_{\chi}^{1}-L_{\kappa}, 0\right)\right)
$$

With $r$ (described earlier), and the threshold $=\rho$, Value at Risk can be calculated as:

$$
\operatorname{VAR}(\rho)=\inf \left\{L_{\kappa}^{*} \mid F_{L_{K}}\left(L_{\kappa}^{*}\right)<1-\rho \%\right\}
$$

and the:

$$
C T E(\rho)=E\left(L_{\kappa} \mid L_{\kappa} \geq \operatorname{VaR}(\rho)\right)
$$

With the VaR and CTE risk proxies, we can calculate the risk margin ratio $=\delta(L)$ with the Solvency 2 Directive percentile method:

$$
\delta(L)=\frac{\rho(L)-E(L)}{E(L)}
$$

where $\rho(L)$ represents VaR and CTE risk measures, and $E(L)$ the best estimate. If this is considered with an assumption that losses would be larger than ransoms: $\propto$ of losses $\leq \propto$ of ransoms. Then the power-law distribution can be calculated with the equation:

$$
P(x)=\propto X^{-\alpha}
$$

where the variance analysis of $\alpha$ parameter is $1<\alpha<2$, with infinite mean and average even when $2<\alpha<3$.

\section{Cognitive design}

Connecting the lost exposure of cyber risk from humancomputer interaction (frequency), in different information knowledge management systems (magnitude), with artificial intelligence, can provide predictive feedback sensors for primary and secondary loss (vulnerabilities). These feedback sensors represent dynamic real time data mechanisms that assist and enable better understanding of the vulnerabilities prior to cyber-attacks. The reliability of cyber risk analytics could increase significantly if decisionmakers have a dynamic and self-adopting AI enhanced feedback sensors to assess, predict, analyse and address the economic risks of cyberattacks.

The survey (in Table 1) identified all relevant AI algorithms, and the mathematical formulae (results in Table 2) articulates some of the possible solutions for the role of these algorithms in designing dynamic automated predictive feedback cognitive system, supported with real-time intelligence.

Cyber risk analytic approaches with dynamic real-time and $\mathrm{AI} / \mathrm{ML}$ self-adapting enhanced technologies that enable predictive risk analytics are identified in Table 1. While the design of a predictive cyber risk analytics is based on confidence intervals and time bound ranges in Table 2. In doing this work we are acutely aware that adding automation and further coupling to a distributed system also brings new opportunities for cascading effects and exposing new attack surfaces. These concerns are fundamental in the areas with increased automation of processes which have classically required human interaction.

\section{Dynamic and self-adapting predictive data analytics with the mathematical formulae}

A range of data sources was used to apply data analytics with the new mathematical formulae. The Comprehensive Threat Intelligence was used to collect data from vulnerability reports and zero-day reports (Cisco 2020). The Chronology of Data Breaches (Land et al. 2020) was used to gather larger sample size from thousands of records collected over the last 10 years (2010-2020). The SonicWall cyber threat report was used to collect probabilistic data on trends of IoT attacks (SonicWall 2019). The aggregate cyber risk from a large sample population is not the ideal measure for calculating the cyber risk of a small and/or medium sized enterprise. Hence, we divided the large sample into subsamples that follow a Poisson distribution with smaller total risk $\lambda_{c}$, where:

$$
\lambda_{c}=\lambda_{c 1}+\ldots+\lambda_{c m}
$$

and $\lambda_{c i}, i=1, \ldots m$ represented as the individual risk of a subsample. Finally, the total cyber risk of the adjusted proportion parameter $p$ is equal to $\lambda_{c}=p \lambda_{c}$. We estimate risk exposure of total IoT cyber risk $p_{1}$ and the IoT cyber risk from nonrecorded devices as $p_{2}$ where: 
Table 2 Dynamic and self-adapting predictive cyber risk analytics based on different levels of cyber risk intelligence

\begin{tabular}{|c|c|c|c|c|c|}
\hline \multirow[t]{2}{*}{ Risk calculation metrics } & \multirow[t]{2}{*}{ Cyber risk } & \multicolumn{4}{|c|}{ IoT cyber risk } \\
\hline & & $\begin{array}{l}\text { Guarded } \\
\text { (Green) }\end{array}$ & $\begin{array}{l}\text { Elevated } \\
\text { (Yellow) }\end{array}$ & High (Amber) & Severe (Red) \\
\hline$E\left(P_{\chi}^{1}\right)$ & $9,225,798$ & $8,302,872$ & $8,323,645$ & $8,495,883$ & $8,826,248.5$ \\
\hline $\operatorname{Prob}\left(\kappa \times \pi_{\chi}^{1} \leq L_{\kappa}\right)$ & $0.362 \%$ & $0.390 \%$ & $0.717 \%$ & $2.952 \%$ & $4.359 \%$ \\
\hline$E\left(\max \left(\kappa \times \pi_{\chi}^{1}-L_{\kappa}, 0\right)\right)$ & 776 & 783 & 31,660 & 281,340 & 807,491 \\
\hline $\operatorname{VAR}(.90)$ & $9,659,815$ & $8,696,453.5$ & $8,706,061$ & $8,797,711.5$ & $8,947,727$ \\
\hline $\operatorname{VAR}(.95)$ & $9,785,002.5$ & $8,807,375.5$ & $8,823,096.5$ & $9,020,017.5$ & $9,510,794.5$ \\
\hline $\operatorname{VAR}(.99)$ & $10,020,820.5$ & $9,031,839.5$ & $9,092,977.5$ & $11,314,474.5$ & $14,126,426.5$ \\
\hline CTE(.90) & $9,823,671.5$ & $8,844,958.5$ & $9,021,798$ & $10,482,011.5$ & $13,461,685$ \\
\hline CTE (.95) & $9,930,712$ & $8,942,115.5$ & $9,283,971$ & $12,076,385$ & $17,770,438$ \\
\hline CTE(.99) & $10,139,834$ & $9,137,848.5$ & $10,729,379.5$ & $22,130,721.5$ & $45,991,022$ \\
\hline
\end{tabular}

$\mathrm{E}\left(\mathrm{P}_{\mathrm{x}}^{1}\right)=$ Expected present value of the loss;

$\operatorname{Prob}\left(\kappa \times \pi_{\chi}^{1} \leq \mathrm{L}_{\kappa}\right)=\operatorname{Prob}($ Shortfall) or Shortfall probability;

$\mathrm{E}\left(\max \left(\kappa \times \pi_{\chi}^{1}-\mathrm{L}_{\kappa}, 0\right)\right)=\mathrm{E}($ Shortfall $)$ or Shortfall of expected present value of the loss;

$\operatorname{VAR}(\rho)=$ Value at Risk;

$\operatorname{CTE}(\rho)=$ Conditional Tail Expectation

Note: Assuming $\mathrm{N}=1000, \mathrm{~b}=1000, \mathrm{r}=0.03, \alpha=0.9, \delta=0.1$ in the IoT cyber risk calculation and number of repetitions $=100,000$

$$
p=p_{1} \times\left(m \times p_{2}^{-1}\right)
$$

To demonstrate how these models can be applied for numerical results, in this section we present a demonstration project of different numerical results. For the numerical estimates in Table 2, we generate 100,000 Monte Carlo simulation runs, for 10,000 IoT devices being hacked. We use these Monte Carlo simulation runs to compare the effectiveness of different risk measures to quantify cyber risk, using the estimate of aggregate distribution of total loses (primary and secondary). We assume that by understanding the cyber risk, we can lower the frequency of cyber breaches $(\alpha=0.9)$. In the next paragraph, we detail our data sources, and how we obtained the required data to present the demonstration of numerical results in Table 2. In the demonstration project, we adopted the numerical results to compare the estimated risk measures under different risk levels: Guarded (Green); Elevated (Yellow); High (Amber); Severe (Red).

$m=$ independent sample size. If we assume that $m=10,000$ and $p=0.00002$. The $p=0.00002$ derived from the findings that IoT devices are attacked within 5 min of being connected to the internet (NetScouts 2018), while over $50 \%$ of the cyber risk professionals do not keep inventory of IoT devices installed (SFG 2017), hence there are potentially over $50 \%$ more IoT devices exposed to attacks. This is calculated as
365 (days in a year) $\times 24$ (hours per day) $\times 60$ (minutes per hour) $-50 \%$ (the cyber-attacks on not recorded IoT devices) ${ }^{1}$ where ten times $p=$ 'high (amber)' risk, twenty times $p=$ 'severe (red)' risk. This reflects on findings that IoT will increase at a rate of 152,200 devices per minute by 2025 (Rosen $2015) \times 525,600$ (minutes in 365 days $)=80$ billion new IoT devices connected annually. This will increase the overall IoT cyber risk level. The twenty times assumption is based on the SonicWall report (SonicWall 2019) stating that IoT malware attacks increased by $215.7 \%$ from $10.3 \mathrm{~m}$ in 2017 to $32.7 \mathrm{~m}$ in 2018 and the trend continued in $2019 .^{2}$ The twenty times assumption represents .99 in Table 1. The corresponding .95 and .90 derive from the .99 calculation. We can also realistically assume that 'guarded (green)' level of cyber maturity would lower the $\alpha=.90$, then we can calculate the shortfall probability, expected shortfall, VaR and CTE for different cyber risk levels and tail risk under different assumptions in Table 1.

While existing cyber risk assessment models are based on individual risk calculation metrics, the approach presented in the mathematical formulae and demonstrated in Table 1, is based on multiple numerical risk metrics. The quantitative

\footnotetext{
$\overline{{ }^{1} 365 \times 24 \times 60 \times .5}=262,800 \div 5=52,560 \div 262,800=0.2 \div 10,000$

${ }^{2}$ SonicWall report (SonicWall 2019) captured real-world data from more than one million sensors in over 215 countries with over 140,000 malware samples collected daily.
} 
approach of the mathematical formulae, when integrated with Excel Macros, presents risk categorisations (Table 1) that are supported with real time intelligence. This presents a dynamic and self-adopting predictive cyber risk analytics approach, that is compliant with the existing NIST 'traffic lights' risk categorisations. The quantitative approach also correlates the NIST standards with the FAIR Institute efforts for quantitative cyber risk analytics. The mathematical formulae is similar to the FAIR-U approach (FAIR 2020), but instead of relying on a specific risk metric, its reliant on multiple numerical risk metrics. For comparison, the mathematical formulae uses different tail risk measurement and compares the impact of cyber risk under different risk categories. The 'high (amber)' and 'severe (red)' risk categories derive numerical representation of how rare and extreme events (black swan events) can increase the cyber risk impact. The impact of risk in VaR (.90) and CTE (.90) is not significant, but the risk margin ration increases significantly when compared to $\operatorname{VaR}(.99)$ and CTE (.99). This provides a quantitative perspective of impact from 'black swan' events, and enables more informative decision making on implementation of low cost and low security vs higher cost and higher security IoT systems, while putting 'black swan' events in IoT risk perspective. Worth noting that although IoT devices today are attacked within 5 min of being connected to the internet (NetScouts 2018), computers connected to internet even back in 2007 were attacked on average every 39 s (Cukier 2007). Given that computers even in 2007 had much more computing power than most IoT devices today, we can anticipate a continuous increase in attack frequency on IoT devices. Although such assumptions given the lack of data can only be described as super forecasting, the estimated average attack detection and mitigation in terms of the 5 min from connection to attack timeframe, can be described with different ML algorithms. Comparing the average attack detection and mitigation time in Fig. 1. with multiple algorithms (Nguyen et al. 2019), including Distributed Edgebased Defence (DED), Centralised Fog-based Defence (CFD), Centralised Fog and Cloud-based Defence (CFCD) and SeArch architecture.

The average attack detection and mitigation time Fig. 1, shows that although some of the NIDS described earlier, can detect IoT attacks within the 5 min average attack time from the moment of connection, none of the NIDS shown in Fig. 1 can mitigate IoT attacks instantly. Therefore, understanding the risks before they occur is of a significant relevance to preventing severe impact from IoT attacks.

\section{Conclusion}

This study reviewed how different AI methods can be applied for cyber risk analytics in extreme environments, such as exploration of outer space, where AI would need to perform all

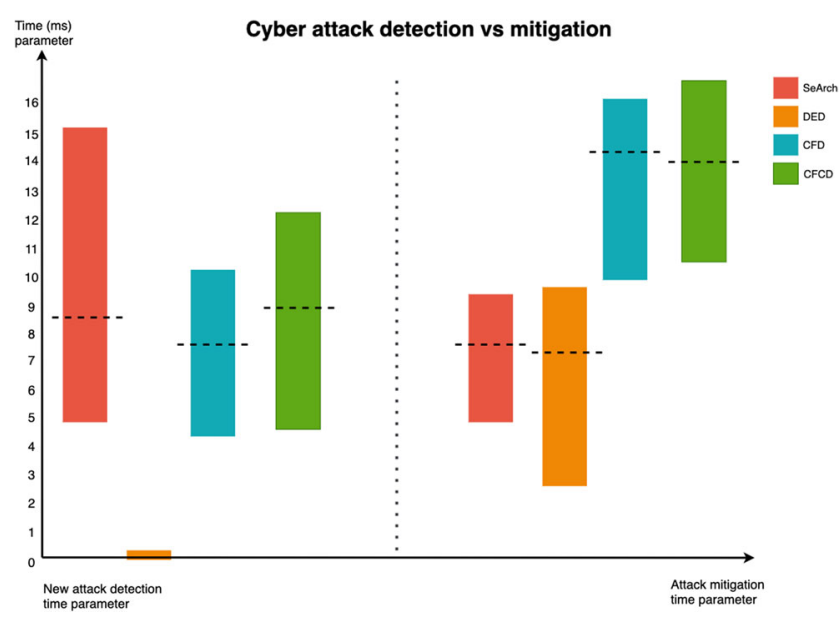

Fig. 1 The average attack detection and mitigation on IoT connected devices

the tasks, including its own risk assessment. The review confirms that for the integration of $\mathrm{AI} / \mathrm{ML}$ in risk analytics, we need to adapt the data strategies to collect the appropriate cyber-risk data. With the integration of IoT systems, new types of data streams are becoming available. Such data streams can be collected and analysed with AI/ML algorithms. The survey review in this paper, identified some of the potential impact assessment approaches that can be redesigned for predictive, dynamic and self-adopting cyber risk analytics. The conclusion builds upon the existing approach for categorising (pooling) risk, but presents a quantitative version of the NIST 'traffic lights' system (demonstrated in Table 2), enhanced with multiple risk calculation metrics that calculate the shortfall probability, expected shortfall, VaR and CTE for different cyber risk levels and tail risk under different assumptions (see Table 2).

The mathematical formulae present a better understanding of the cost and risk evaluation with multiple risk calculation metrics for different cyber risk levels and tail risk under different assumptions. The value of safety and cyber risk in extreme environments - such as outer space, can be explained in economic terms, where the level of cybersecurity is based on the risk acceptance level and the co-ordination of sufficient protection of the communications networks.

This study presents a mathematical formula for the future cyber risk developments that are reshaping the data analytics of supply and control systems. The mathematical formulae represent an advancement and integration of the NIST 'traffic lights' system and the FAIR-U Tool, though 'pooling' of risk data into calculation metrics, while anonymising data from individual IoT devices.

Secondly, the co-ordination of supply and control systems cyber protection though $\mathrm{AI} / \mathrm{ML}$ must be reliable to prevent abuse from the AI itself. The mathematical formulae in this article relies on multiple risk calculation metrics, while existing cyber risk assessment approached are designed with 
individual risk calculation metrics. The integration of multiple risk metrics presents a more robust protection from abuse of individual data intelligence streams.

Thirdly, the predictive cyber risk analytics as presented in the article, are based on different levels of risk intelligence that are 'pooled' into numbers and not presented as individual risk events. Hence, it allows for anonymising the risk data, and after applying the mathematical model, the data is presented into anonymous risk categories.

\section{Limitations and further research}

$\mathrm{AI} / \mathrm{ML}$ in cyber risk data analytics integrated in the supply chains and control systems would present innovative and cost-effective ways to protect such data. In addition, the AI/ML analysis of the threat event frequency, with a dynamic and self-adopting AI enhanced methodology, would empower the design of a cognition engine mechanisms for predicting the loss magnitude through the control, analysis, distribution and management of probabilistic data. The development of such cognitive engine and its application, would undoubtedly bring multiple benefits and would enable deeper understanding of the impact of cyber risk at the edge. Nonetheless, IoT networks represent complex coupled systems (D. De Roure et al. 2019), that can be described as cyber-physical social machines (Madaan et al. 2018) and social machines (David De Roure et al. 2015) should be observed in practice (Shadbolt et al. 2019). Given that IoT is considered as critical enabler (Lee et al. 2019a) of value creation (Lee et al. 2019b), the findings of this study would probably be best verified when observed in practice.

Acknowledgments Eternal gratitude to the Fulbright Scholar Project.

\section{Availability of data and material Not applicable}

Authors' contributions Dr. Petar Radanliev: main author; Prof. Dave De Roure: supervision; Dr. Kevin Page: supervision; Dr. Max Van Kleek, Omar Santos, La'Treall Maddox, Prof. Pete Burnap, and Prof. Carsten Maple: supervision and corrections.

Funding This work was funded by the UK EPSRC [grant number: EP/ S035362/1] and by the Cisco Research Centre [grant number 1525381].

\section{Compliance with ethical standards}

Conflicts of interest/competing interests The authors declare that they have no conflicting and/or competing interests.

\section{Code availability Not applicable}

Open Access This article is licensed under a Creative Commons Attribution 4.0 International License, which permits use, sharing, adaptation, distribution and reproduction in any medium or format, as long as you give appropriate credit to the original author(s) and the source, provide a link to the Creative Commons licence, and indicate if changes were made. The images or other third party material in this article are included in the article's Creative Commons licence, unless indicated otherwise in a credit line to the material. If material is not included in the article's Creative Commons licence and your intended use is not permitted by statutory regulation or exceeds the permitted use, you will need to obtain permission directly from the copyright holder. To view a copy of this licence, visit http://creativecommons.org/licenses/by/4.0/.

\section{References}

Abegunde, J., Xiao, H., \& Spring, J. (2016) A dynamic game with adaptive strategies for IEEE 802.15.4 and IoT. 2016 IEEE Trustcom/ BigDataSE/ISPA, 473-480. https://doi.org/10.1109/TrustCom. 2016.0099

Agyepong E, Cherdantseva Y, Reinecke P, Burnap P (2019) Challenges and performance metrics for security operations center analysts: a systematic review. J Cyber Secur Technol 4(1):1-28. https://doi.org/ 10.1080/23742917.2019.1698178

Al-Turjman F (2020) Intelligence and security in big 5G-oriented IoNT: an overview. Futur Gener Comput Syst 102:357-368. https://doi. org/10.1016/j.future.2019.08.009

Anagnostopoulos, C., \& Hadjiefthymiades, S. (2019) A Spatio-temporal data imputation model for supporting analytics at the edge. Digital transformation for a sustainable society in the 21 st century: 18th IFIP WG 6.11 conference on E-Business, E-Services, and ESociety, I3E 2019, Trondheim, Norway, September 18-20, 2019, Proceedings, 11701, 138

Anthi E, Williams L, Burnap P (2018) Pulse: an adaptive intrusion detection for the internet of things. Living in the Internet of Things: cybersecurity of the IoT. 35:1-4. https://doi.org/10.1049/cp.2018. 0035

Anthi E, Williams L, Slowinska M, Theodorakopoulos G, Burnap P (2019) A supervised intrusion detection system for smart home IoT devices. IEEE Internet Things J 6(5):9042-9053. https://doi. org/10.1109/JIOT.2019.2926365

Anthonysamy, P., Rashid, A., \& Chitchyan, R. (2017) Privacy requirements: present \& future. 2017 IEEE/ACM 39th international conference on software engineering: software engineering in society track (ICSE-SEIS), 13-22. https://doi.org/10.1109/ICSE-SEIS. 2017.3

Barker K, Lambert JH, Zobel CW, Tapia AH, Ramirez-Marquez JE, Albert L, Nicholson CD, Caragea C (2017) Defining resilience analytics for interdependent cyber-physical-social networks. Sustain Resilient Infrastruct 2(2):59-67. https://doi.org/10.1080/23789689. 2017.1294859

Bashir H, Lee S, Kim KH (2019) Resource allocation through logistic regression and multicriteria decision making method in IoT fog computing. Trans Emerg Telecommun Technol. https://doi.org/10. 1002/ett.3824

Berman D, Buczak A, Chavis J, Corbett C (2019) A survey of deep learning methods for cyber security. Information 10(4):122. https://doi.org/10.3390/info10040122

Blanco-Filgueira B, Garcia-Lesta D, Fernandez-Sanjurjo M, Brea VM, Lopez P (2019) Deep learning-based multiple object visual tracking on embedded system for IoT and mobile edge computing applications. IEEE Internet Things J 6(3):5423-5431. https://doi.org/10. 1109/JIOT.2019.2902141

Boyes H, Hallaq B, Cunningham J, Watson T (2018) The industrial internet of things (IIoT): an analysis framework. Comput Ind 101: 1-12. https://doi.org/10.1016/J.COMPIND.2018.04.015 
Breza M, Tomic I, McCann J (2018) Failures from the environment, a report on the first FAILSAFE workshop. ACM SIGCOMM Comput Commun Rev 48(2):40-45. https://doi.org/10.1145/ 3213232.3213238

Bui KHN, Jung JE, Camacho D (2017) Game theoretic approach on realtime decision making for IoT-based traffic light control. Concurr Comput 29(11):e4077. https://doi.org/10.1002/cpe.4077

Buith, J (2016) Cyber value at risk in the Netherlands. https://www2. deloitte.com/content/dam/Deloitte/nl/Documents/financial-services/ deloitte-nl-fsi-cyber-value-at-risk.pdf

Cao, B., Zhang, L., Li, Y., Feng, D., \& Cao, W. (2019) Intelligent offloading in multi-access edge computing: a state-of-the-art review and framework. In: IEEE communications magazine. Institute of Electrical and Electronics Engineers Inc., (vol. 57, issue 3, pp. 5662). https://doi.org/10.1109/MCOM.2019.1800608

Charpentier, A. (2014) Computational actuarial science with R. In: Computational actuarial science with R Chapman and Hall/CRC. https://doi.org/10.1201/b17230

Cisco (2020) Cisco Talos Intelligence Group. Comprehensive threat intelligence. https://talosintelligence.com/

Craggs, B., \& Rashid, A. (2017) Smart cyber-physical systems: beyond usable security to security ergonomics by design. 2017 IEEE/ACM 3rd international workshop on software engineering for smart cyberphysical systems (SEsCPS), 22-25. https://doi.org/10.1109/ SEsCPS.2017.5

Cui Q, Gong Z, Ni W, Hou Y, Chen X, Tao X, Zhang P (2019) Stochastic online learning for mobile edge computing: learning from changes. IEEE Commun Mag 57(3):63-69. https://doi.org/10.1109/MCOM. 2019.1800644

Cukier, M. (2007) Study: hackers attack every 39 seconds $\mid$ A. James Clark School of Engineering, University of Maryland. University of Maryland. https://eng.umd.edu/news/story/study-hackers-attackevery-39-seconds

Davis M, Gilbert M, Simon K, Stephen M, Gilibrays Ocen G (2019) State of cyber security: the Ugandan perspective. Int J Sci Eng Res 10(4): 713-724 http://www.ijser.org

De Roure, David, Hooper, C., Page, K., Tarte, S., \& Willcox, P. (2015) Observing social machines part 2. Proceedings of the ACM web science conference on ZZZ - WebSci'15, 1-5. 1145/2786451.2786475

De Roure, D., Page, K. R., Radanliev, P., \& Van Kleek, M. (2019) Complex coupling in cyber-physical systems and the threats of fake data. Living in the Internet of Things (IoT 2019), 2019 Page, 11 (6 pp.). https://doi.org/10.1049/cp.2019.0136

Diro AA, Chilamkurti N (2018) Distributed attack detection scheme using deep learning approach for Internet of Things. Futur Gener Comput Syst 82:761-768. https://doi.org/10.1016/j.future.2017.08. 043

FAIR (2017) What is a cyber value-at-risk model? http://www. fairinstitute.org/blog/what-is-a-cyber-value-at-risk-model

FAIR (2020) FAIR risk analytics platform management. FAIR-U model. https://www.fairinstitute.org/fair-u

Gebremariam, A. A., Usman, M., \& Qaraqe, M. (2019) Applications of artificial intelligence and machine learning in the area of SDN and NFV: a survey. 16th international multi-conference on systems, signals and devices, SSD 2019, 545-549. https://doi.org/10.1109/SSD. 2019.8893244

Ghirardello, K., Maple, C., Ng, D., \& Kearney, P. (2018) Cyber security of smart homes: development of a reference architecture for attack surface analysis. Living in the Internet of Things: Cybersecurity of the IoT - 2018, 45 (10 pp.) 45 (10 pp.). https://doi.org/10.1049/cp. 2018.0045

Guo Y, Cao H, Han S, Sun Y, Bai Y (2018) Spectral-spatial hyperspectral image classification with $\mathrm{K}$-nearest neighbor and guided filter. IEEE Access 6:18582-18591. https://doi.org/10.1109/ACCESS.2018. 2820043
Hu R, Wen S, Zeng Z, Huang T (2017) A short-term power load forecasting model based on the generalized regression neural network with decreasing step fruit fly optimization algorithm. Neurocomputing 221:24-31. https://doi.org/10.1016/j.neucom. 2016.09.027

Khan F (2020) Safety and integrity management of operations in harsh environments. Saf Extreme Environ 2(1):1-2. https://doi.org/10. 1007/s42797-020-00019-5

Land, M., Mok, B., Roane, E., \& Stephens, P. (2020) Privacy rights clearinghouse. Chronology of data breaches. https://privacyrights. org/data-breaches

Lee, B., Cooper, R., Hands, D., \& Coulton, P. (2019a) Design drivers: a critical enabler to meditate value over the NPD process within internet of things. $4 \mathrm{~d}$ conference proceedings: meanings of design in the next Era. Osaka: DML (Design Management Lab), Ritsumeikan University, 96-107

Lee, B., Cooper, R., Hands, D., \& Coulton, P. (2019b) Value creation for IoT: challenges and opportunities within the design and development process. Living in the Internet of Things (IoT 2019). IET, living in the Internet of Things 2019, London, UK, 1-8. https:// doi.org/10.1049/cp.2019.0127

Li H, Ota K, Dong M (2018) Learning IoT in edge: deep learning for the Internet of Things with edge computing. IEEE Netw 32(1):96-101. https://doi.org/10.1109/MNET.2018.1700202

Madaan A, Nurse J, de Roure D, O’Hara K, Hall W, Creese S (2018) A storm in an IoT cup: the emergence of cyber-physical social machines. SSRN Electron J. https://doi.org/10.2139/ssrn.3250383

Malhotra Y (2018) Cognitive computing for anticipatory risk analytics in intelligence, surveillance, \& reconnaissance (ISR): model risk management in artificial intelligence \& machine learning (presentation slides). SSRN Electron J. https://doi.org/10.2139/ssrn.3111837

Maple C (2017) Security and privacy in the internet of things. J Cyber Policy 2(2):155-184. https://doi.org/10.1080/23738871.2017. 1366536

Moura J, Hutchison D (2019) Game theory for multi-access edge computing: survey, use cases, and future trends. IEEE Commun Surv Tutorials 21(1):260-288. https://doi.org/10.1109/COMST.2018. 2863030

NetScouts (2018) Dawn of the TerrorBIT era NETSCOUT threat intelligence report-powered by ATLAS findings from second half 2018. https://www.netscout.com/sites/default/files/2019-02/SECR 001 EN-1901 - NETSCOUT threat intelligence report 2H 2018.pdf

Nguyen TG, Phan TV, Nguyen BT, So-In C, Baig ZA, Sanguanpong S (2019) SeArch: a collaborative and intelligent NIDS architecture for SDN-based cloud IoT networks. IEEE Access 7:107678-107694. https://doi.org/10.1109/ACCESS.2019.2932438

Park D, Kim S, An Y, Jung J-Y (2018) LiReD: a light-weight real-time fault detection system for edge computing using LSTM recurrent neural networks. Sensors 18(7):2110. https://doi.org/10.3390/ s18072110

Porambage, P., Kumar, T., Liyanage, M., Partala, J., Lovén, L., Ylianttila, M., \& Seppänen, T. (2019) Sec-edgeAI: AI for edge security vs security for edge AI BrainICU-measuring brain function during intensive care view project ECG-based emotion recognition view project Sec-EdgeAI: AI for edge security vs security for edge AI. https://www.researchgate.net/publication/330838792

Radanliev P, De Roure D, Nicolescu R, Huth M, Montalvo RM, Cannady S, Burnap P (2018) Future developments in cyber risk assessment for the internet of things. Comput Ind 102:14-22. https://doi.org/10. 1016/J.COMPIND.2018.08.002

Radanliev P, De Roure D, Nurse JRC, Mantilla Montalvo R, Cannady S, Santos O, Maddox L, Burnap P, Maple C (2020a) Future developments in standardisation of cyber risk in the Internet of Things (IoT). SN Appl Sci 2(2):1-16. https://doi.org/10.1007/s42452-019-1931-0

Radanliev P, De Roure D, Van Kleek M, Ani U, Burnap P, Anthi E, Nurse JRC, Santos O, Montalvo RM, Maddox LT (2020b) 
Dynamic real-time risk analytics of uncontrollable states in complex internet of things systems: cyber risk at the edge. Environ Syst Decisions 1:1-12. https://doi.org/10.1007/s10669-020-09792-x

Radanliev P, De Roure D, Van Kleek M, Santos O, Ani U (2020c) Artificial intelligence in cyber physical systems. AI Soc 1:1-14. https://doi.org/10.1007/s00146-020-01049-0

Radanliev P, De Roure D, Walton R, Van Kleek M, Montalvo RM, Maddox L, Santos O, Burnap P, Anthi E (2020d) Artificial intelligence and machine learning in dynamic cyber risk analytics at the edge. SN Appl Sci 2(11):1-8. https://doi.org/10.1007/s42452-02003559-4

Radanliev P, De Roure D, Page K, Nurse JRC, Montalvo RM, Santos O, Maddox L, Burnap P (2020e) Cyber risk at the edge: current and future trends on cyber risk analytics and artificial intelligence in the industrial internet of things and industry 4.0 supply chains. Cybersecurity, Springer Nat 3(13):1-21. https://doi.org/10.1186/ s42400-020-00052-8

Roopak, M., Yun Tian, G., \& Chambers, J. (2019) Deep learning models for cyber security in IoT networks. 2019 IEEE 9th annual computing and communication workshop and conference, CCWC 2019, 452 457. https://doi.org/10.1109/CCWC.2019.8666588

Rosen, M. (2015) Driving the digital agenda requires strategic architecture

Safa NS, Maple C, Watson T, Von Solms R (2018) Motivation and opportunity based model to reduce information security insider threats in organisations. J Inf Secur Appl 40:247-257. https://doi. org/10.1016/J.JISA.2017.11.001

Sangaiah AK, Medhane DV, Han T, Hossain MS, Muhammad G (2019) Enforcing position-based confidentiality with machine learning paradigm through mobile edge computing in real-time industrial informatics. IEEE Trans Ind Inform 15(7):4189-4196. https://doi.org/10. 1109/TII.2019.2898174

SFG. (2017) The Internet of Things (IoT): a new era of third-party risk shared assessments. https://sharedassessments.org/the-internet-ofthings/

Shadbolt N, O'Hara K, De Roure D, Hall W (2019) The theory and practice of social machines. Springer Int Publ. https://doi.org/10. 1007/978-3-030-10889-2

SonicWall (2019) Mid-year update: 2019 sonicwall cyber threat report. https:/i.crn.com/sites/default/files/ckfinderimages/userfiles/images/ crn/custom/2019/SonicWall Cyber Threat Report-Mid-Year Update - 2019.pdf

Sultana N, Chilamkurti N, Peng W, Alhadad R (2019) Survey on SDN based network intrusion detection system using machine learning approaches. Peer-to-Peer Netw Appl 12(2):493-501. https://doi. org/10.1007/s12083-017-0630-0

Sun, D., Wu, Z., Wang, Y., Lv, Q., \& Hu, B. (2019) Risk prediction for imbalanced data in cyber security: a Siamese network-based deep learning classification framework. Proceedings of the international joint conference on neural networks, 2019-July, 1-8. https://doi.org/ 10.1109/IJCNN.2019.8852030

Syafrudin M, Fitriyani N, Alfian G, Rhee J (2018) An affordable fast early warning system for edge computing in assembly line. Appl Sci 9(1):84. https://doi.org/10.3390/app9010084

Tanczer, L. M., Steenmans, I., Elsden, M., Blackstock, J., \& Carr, M. (2018) Emerging risks in the IoT ecosystem: who's afraid of the big bad smart fridge? Living in the Internet of Things: cybersecurity of the IoT, 33 (9 pp.). https://doi.org/10.1049/cp.2018.0033

Ullah I, Ahmad S, Mehmood F, Kim D (2019) Cloud based IoT network virtualization for supporting dynamic connectivity among connected devices. Electronics 8(7):742. https://doi.org/10.3390/ electronics 8070742

Van Kleek, M., Binns, R., Zhao, J., Slack, A., Lee, S., Ottewell, D., \& Shadbolt, N. (2018) X-ray refine. Proceedings of the 2018 CHI conference on human factors in computing systems - CHI ' $18,1-$ 13. https://doi.org/10.1145/3173574.3173967

Vinayakumar R, Alazab M, Soman KP, Poornachandran P, Al-Nemrat A, Venkatraman S (2019) Deep learning approach for intelligent intrusion detection system. IEEE Access 7:41525-41550. https://doi.org/ 10.1109/ACCESS.2019.2895334

Wang J, Hu J, Min G, Zhan W, Ni Q, Georgalas N (2019a) Computation offloading in multi-access edge computing using a deep sequential model based on reinforcement learning. IEEE Commun Mag 57(5): 64-69. https://doi.org/10.1109/MCOM.2019.1800971

Wang X, Han Y, Wang C, Zhao Q, Chen X, Chen M (2019b) In-edge AI: intelligentizing mobile edge computing, caching and communication by federated learning. IEEE Netw 33(5):156-165. https://doi. org/10.1109/MNET.2019.1800286

Yin H, Xue M, Xiao Y, Xia K, Yu G (2019) Intrusion detection classification model on an improved k-dependence Bayesian network. IEEE Access 7:157555-157563. https://doi.org/10.1109/ACCESS. 2019.2949890

Zhang, D., Bao, W., Fang, T., Liang, W., Zhou, W., Ma, L., Gao, X., \& Niu, L. (2019) Edge task allocation scheme based on data classification. Proceedings -2019 5th international conference on big data and information analytics, BigDIA 2019, 132-138. https://doi.org/ 10.1109/BigDIA.2019.8802859 